$\$=1$ 原

\title{
Optimum density determination for bouguer correction using statistical methods: a case study from north of Iran
}

\author{
Ata Eshaghzadeh ${ }^{1 *}$, Roghayeh Alsadat Kalantari ${ }^{1}$, Zohreh Moeini Hekmat ${ }^{2}$ \\ ${ }^{1}$ Graduate student of geophysics, Institute of Geophysics, University of Tehran, Iran \\ ${ }^{2}$ Graduate student of geophysics, Islamic Azad University, Hamedan,Iran \\ *Corresponding author E-mail: eshagh@ut.ac.ir
}

\begin{abstract}
The main aim of initial gravity data processing is to determine the density of under-research geological structures and stratification mat rials in this case. The density is important for the calculation of the Bouguer plate and terrain corrections. To achieve the corrected gravity data with high quality and accuracy, exact estimation of the density is very significant, but representative optimum density value for an area of interest is notoriously difficult to obtain. In this paper, several statistical methods based on the correlation are proposed, such as variation and fractal for surface optimum density determination. The efficiency of the methods has been employed for a case study in north of Iran.
\end{abstract}

Keywords: Bouguer; Correlation; Fractal; Optimum Density; Variation.

\section{Introduction}

Density is defined as the mass per unit of volume of a material. It has been measured in different units and different numerical values in the C.G.S and SI systems. An awareness of the value of bulk density is obviously important to gravity data interpretation. The more precise corrected gravity data, the more accurate quantitative and qualitative results are. $0.1 \mathrm{gr} / \mathrm{cm} 3$ in the reduction density, and nearly $0.42 \mathrm{mGal}$ in Bouguer anomaly for per $100 \mathrm{~m}$, is not a great error in itself, but an error of $0.1 \mathrm{~g} / \mathrm{cm} 3$ in the density may have a huge impact on the interpretations of Bouguer anomalies (Yamamoto 1998). The The near-surface density of the material under a hill can be determined by a prevalent method devised by L. Nettleton (1939). Nettleton's method uses a profile measured across a topographic feature to find the best estimate of bulk density for a region. Thereafter, effective and efficient methods for density determinations from surface gravity measurements have been extensively developed (e.g. Parasnis 1952, Rikitake et al. 1965, Fukao et al. 1981, Murata 1993). Parasnis (1972) proposed an analytical method for calculation surface average density. Most of these methods adopted statistical procedures using the correlations among Bouguer anomalies, free-air anomalies, station heights, and reduction density itself from surface gravity data. Nevertheless, the mentioned methods in many cases lose their efficiency especially in flat areas. Several methods have been proposed to estimate a variable density of surface and near-surface topography (Vajk 1956, Grant \& Elsaharty 1962, Bichara \& Lakshmanan 1983, Rimbert et al. 1987, Moribayashi 1990, Murata 1993). Since the residual anomalies after gravity reduction using a single constant density, still contain density inhomogeneities. They applied the method of overlapping windows and determined a Bouguer reduction density for each of the windows using the data within each of them. Density can be measured through vertical boreholes, drilled to explore the nature of an assumed structure.
The resulting density in the borehole is employed to refine the interpretation of the structure. Determined density using borehole seismic velocities, gamma-gamma logging and borehole gravimetry methods can be considered. Verifying and interpretation of borehole gravimetry give a good estimation of bulk in situ rock densities (e.g. Hammer 1950, Gibb \& Thomas 1980, LaFehr 1983) Sissons (1981) configured a least-squares method for the direct inversion of surface and subsurface gravity measurements to estimate in situ rock densities. Thorarinsson and Magnusson (1990) and Chapin (1996) presented fractal analysis methods for Bouguer density determination. However, these methods associated with subsurface gravity measurements are valid only for quite limited area where the rock unit is reasonably homogeneous in composition. A more well-known method is the Nettleton's method that is widely used to obtain an optimal average density value; however, in some of the areas its efficiency reduces, particular the even region. We have exhibited several methods based on statistical techniques and fractal property that restrain its proficiency in many areas with different morphology.

\section{Study region}

The studied region with an area of approximately $2800 \mathrm{~km}^{2}$ is located in the south of the Caspian Sea, Mazandaran province, Iran (Fig.1). The northern part of this area has been covered by the Quaternary sediments such as loose alluvium; shore line sands, fluvial clay plain, young alluvium, silt and clay flats. The sediments and rocks of the geological formations are related to the Neogene and Paleogene era such as silt, marl, silty marly limestone, gypsum, sand stone, silty marl, etc. they have formed the middle and soutern parts of the mentioned zone. The gravity data set consists of 728 measurements dispersed stations. 


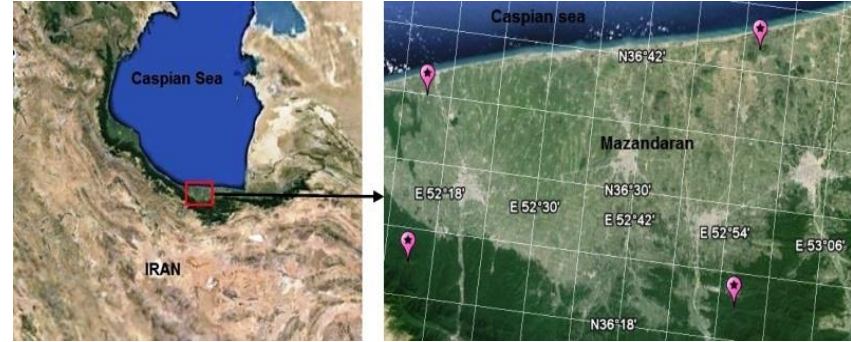

Fig. 1: Situation of the Case Study Region Has Been Determined with the Red Rectangular in the Left Picture. in the Right Picture, the Pink Cursors Display the Quadrangle of the Case Study Region.

\section{Methodology}

This paper comprises the 4 statistical procedures inclusive of the correlation, variations' coefficient, weighted variance and fractal dimension methods. Using this statistical process can compare the comparative intensity of the variability of the various statistic populations. Gravity data is a complex appearance combination of scale-dependent and scale-independent (fractal) components. The gravity effects of geological structures distributions with various densities are essentially scale-dependent, while topography is the primary scale-independent component.

Theoretically, the correlation, variation coefficient and fractal dimension should decrease with increasing density. According to the Bouguer slab formula:

$$
\delta b_{c}=A \rho \times h
$$

\section{That}

$$
A=2 \pi G
$$

Where $\delta b$, is the Bouguer correction and A is the constant 0.0419, for units in meters. In (1), the A $\rho$ term is scale-dependent, while $h$ (elevation) is scale independent.

These techniques are based on computed Bouguer anomalies using different densities. The aim of these methods is finding the density that the final result should be an optimum near-surface density which is the best one that reduces all the data.

\subsection{Correlation method}

Unlike Nettleton's method in which the criterion is minimum correlation between topography and Bouguer anomalies with different densities along a profile, the correlation between the Bouguer anomalies and altitude related to the gravity reading stations while the area thoroughly be covered of sampling points on the ground surface is spotted.

In order to achieve our desirable results, the correlation coefficient is estimated with two vectors:

$$
\operatorname{correl}(x, y)=\frac{\sum_{i=1}^{n}(x-\bar{x})(y-\bar{y})}{\sqrt{\sum_{i=1}^{n}(x-\bar{x})^{2} \sum_{i=1}^{n}(y-\bar{y})^{2}}}
$$

Where $\bar{x}$ and $\bar{y}$ are the average of two numerical sets with $\mathrm{n}$ elements ( $x$ and $y$ are the Bouguer data set, and height data set respectively). The procedure for a given data set is performed as follows. After calculating the Bouguer anomalies with various densities, the correlation between the elevation and Bouguer anomalies of the entire different stations in the study area is computed. The plot of the correlation versus the density for 14 classes of the Bouguer grids computed using different Bouguer densities from the Mazandaran's gravity data set (Fig.2). A straight-line least-squares is fitted to curve. Linear trend is caused by the influ- ence of the linear Bouguer slab equation. According to the straight-line equation, the correlations which rival the densities will be computed. Therefore, elimination of the least-squares linear reply from the curve of the correlation values versus density, yields results that show a unique density that minimizes the topographic effects in Mazandaran region (Fig.3). This value is 2.3 $\mathrm{gr} / \mathrm{cm}^{3}$.

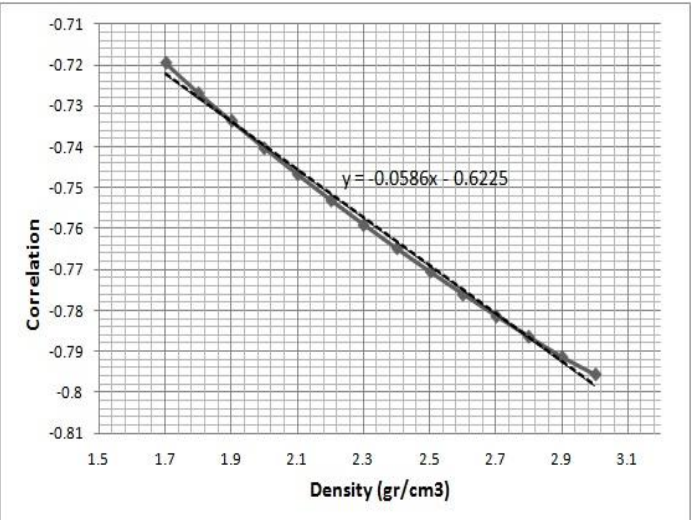

Fig. 2: Plot of the Correlation Versus Density for 14 Classes of the Bouguer Grids Computed Using Different Bouguer Densities from the Mazandaran Gravity Data Set. The Linear Trend Is Caused Made by the Influence of the Linear Bouguer Slab Equation.

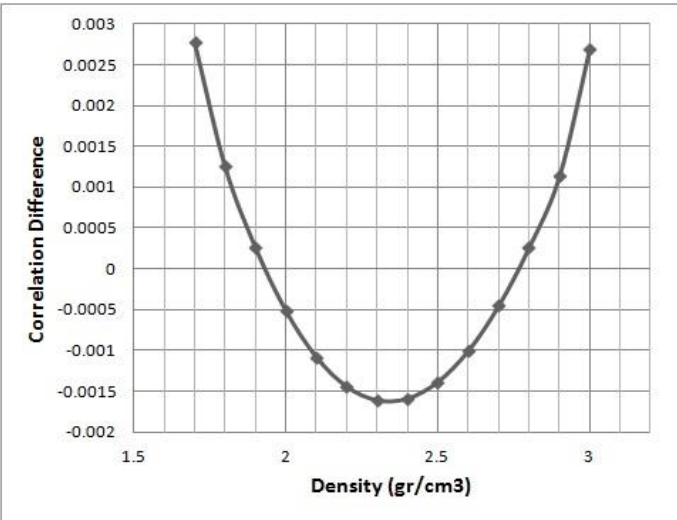

Fig. 3: Plot of the Correlation Differences versus the Density for 14 Classes of Different Bouguer Densities. The Effects of Topography and Bouguer Slab are minimized at $2.3 \mathrm{Gr} / \mathrm{Cm}^{3}$ Densities. In other Word, this Density Is Optimum Density for Bouguer and Terrain Corrections.

\subsection{Variations coefficient method}

The variations coefficient is defined as normalized standard deviation, that is.

$C V=\frac{S}{X}$

Where $\mathrm{S}$ is the standard deviation and $\bar{X}$ is the average of the statistical society.

The variations coefficient related to the Bouguer gravity obtained of the various densities is calculated for the under study area. The drawn curve shows the variation coefficient versus the 14 variant densities (Fig.4) .The crossover points have been determined with orange color. The least-squares line superimpose on variations coefficient - density curve. The difference between the solution, the least-squares line fitted to the variations coefficient, and initial function are displayed in Figure 4, unfolding a function that becomes a minimum at $2.3 \mathrm{gr} / \mathrm{cm}^{3}$ (Fig.5). The result is similar to the correlation method. 


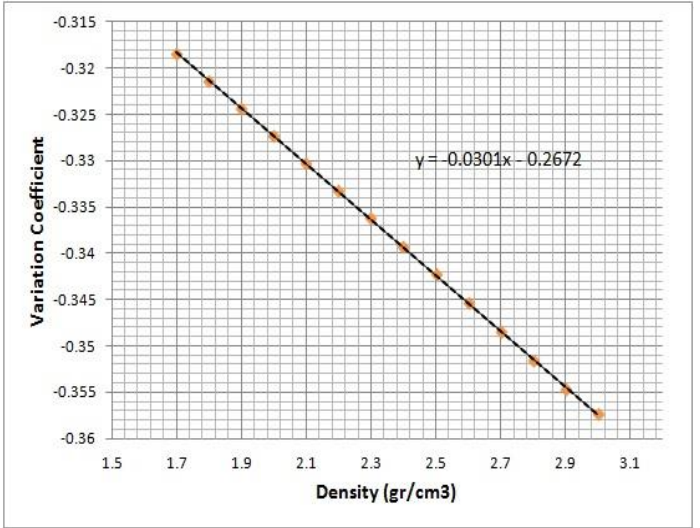

Fig. 4: Plot of the Variation Coefficient Versus Density for 14 Classes of the Bouguer Grids Computed Using Different Bouguer Densities from the Mazandaran Gravity Data Set. The Points Has Been Determined with Orange Color. The Least-Squares Line Superimpose on Variations Coefficient - Dens -Ty Function.

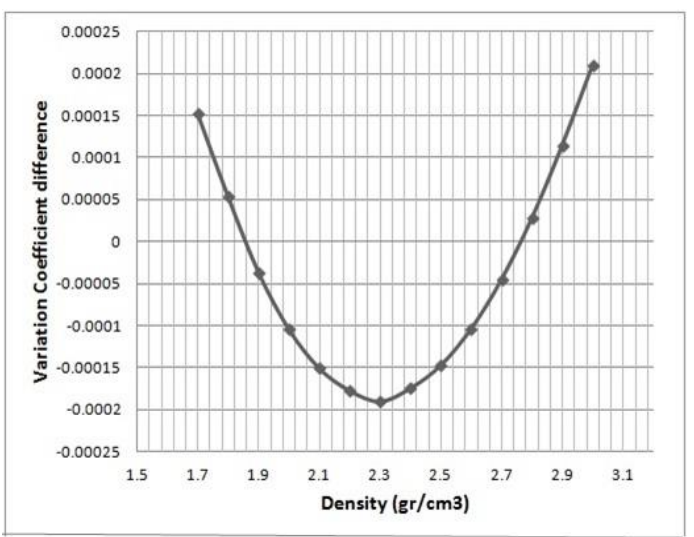

Fig. 5: Plot of the Variation Coefficient Differences versus the Density for 14 Classes of Different Bouguer Densities. The Effects of Topography and Bouguer Slab are minimized at $2.3 \mathrm{Gr} / \mathrm{Cm}^{3}$ Density. In other Word, This Density Is Optimum Density for Bouguer and Terrain Corrections.

\subsection{Weighted variance method}

Sampling is performed from the obtained Bouguer anomaly grid of the density $\rho_{\mathrm{w}}$ with intervals $\mathrm{d}_{1}, \mathrm{~d}_{2} \ldots \mathrm{d}_{\mathrm{i}}$. Afterwards, the variance will be computed for each generated data set. The weighted variance is given as:

$$
\lambda_{w}=\frac{\left(n_{1}-1\right) \lambda_{1}+\left(n_{2}-1\right) \lambda_{2}+\ldots+\left(n_{i}-1\right) \lambda_{i}}{\left(n_{1}-1\right)+\left(n_{2}-1\right)+\ldots+\left(n_{i}-1\right)}
$$

Where $\lambda_{\mathrm{w}}$ is the weighted variance, $\mathrm{n}_{1}$ and $\lambda_{1}$ are the number and variance of data set by sampling with length $d_{1}, n_{2}$ and $\lambda_{2}$ are the number and variance of data set by sampling with length $\mathrm{d}_{2}$ and so forth.

This procedure is done for the Bouguer anomalies with densities $\rho_{1}, \rho_{2} \ldots \rho_{\mathrm{i}}$. Accordingly will be attained the weighted variances $\lambda_{1}$, $\lambda_{2} \ldots \lambda_{\mathrm{i}}$. The curve of the weighted variances versus the 14 variant densities is displayed (Fig.6). The least-squares line is fitted on the curve. The difference between the weighted variances from equation 4 and straight line formula is shown (Fig.7).

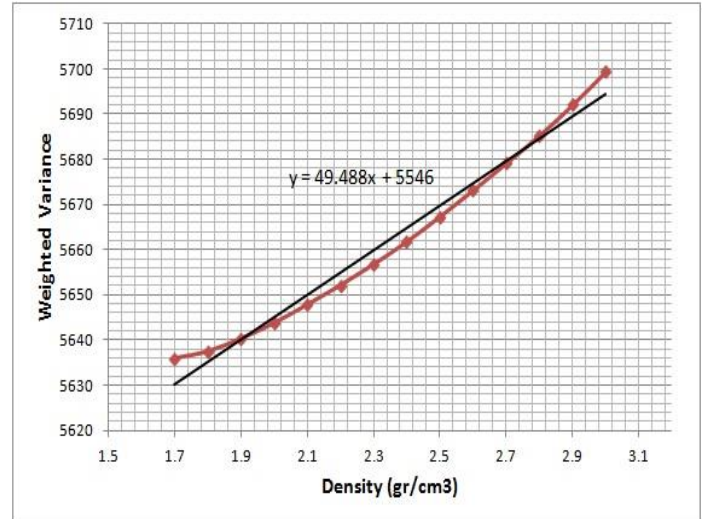

Fig. 6: Plot of the Weighted Variance Versus Density for 14 Classes of the Bouguer Grids Computed Using Different Bouguer Densities from the Mazandaran Gravity Data Set. The Linear Trend Is Caused by the Influence of the Linear Bouguer Slab Equation.

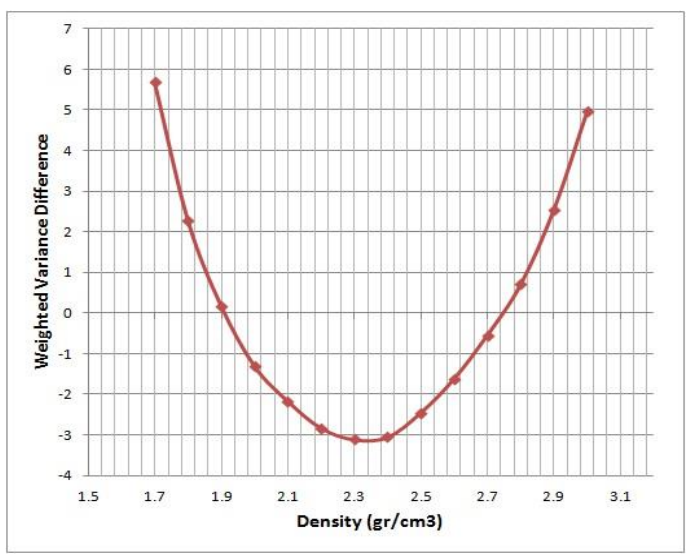

Fig. 7: Plot of the Weighted Variance Differences versus the Density for 14 Classes of Different Bouguer Densities. The Effects of Topography and Bouguer Slab are minimized at $2.3 \mathrm{Gr} / \mathrm{Cm}^{3}$ Density. In other Word, this Density Is Optimum Density for Bouguer and Terrain Corrections.

The minimum value of the weighted variance difference is related to density of $2.3 \mathrm{gr} / \mathrm{cm}^{3}$. This density is the ideal Bouguer density for the under study region.

\subsection{Fractal method}

The detectability limits of a large-scale geophysical measuring network such as station spacing in gravity, magnetic depends on the fractal dimension of the network and the anomaly (Lovejoy et al., 1986; Korvin, 1992). The gravity anomaly resulting from the fractal nature of nonrandom sources distribution of density cannot be accurately measured unless its fractal dimension does not exceed the difference of the 2-D Euclidean and fractal dimension of the network (Thorarinsson and Magnusson 1990, Lovejoy et al. 1986, Korvin 1992). The scale-dependence of the A $\rho$ term manifests itself as a purely linear effect. Therefore, like the statistical methods that have been described in previous portions, a difference between the density function (the curve of the fractal dimension versus density), and the least-squares line fitted to the fractal dimension yields results, which show a unique density that minimizes the topographic effects. Briefly, this value represents the best density to use in the Bouguer slab correction.

Resembling the weighted variance method the gravity data sampling is carried out with variant distances for the each Bouguer anomaly with specific density. Then equivalent with the sampling different distances will be the gravity data set. The variance of the data sets is calculated and plotted logarithmically versus the logarithm of the sampling distance value of each class. Then, the plot is checked visually to distinguish whether a least-squares regression line can be fitted to the values, or to some range of the values. The fractal dimension is derived from the slope of the fitted line. 
This scheme is achieved for the computed Bouguer anomalies using diverse densities. The fractal dimension can be estimated as:

$$
D=1+10^{d}
$$

Where $\mathrm{d}$ is the slope of the fitted least squares line and D is the fractal dimension.

Simple Bouguer gravity grids were computed for the data set of Mazandaran region and fractal dimensions determined for densities ranging from $1.7 \mathrm{gr} / \mathrm{cm}^{3}$ through $3.00 \mathrm{gr} / \mathrm{cm}^{3}$ at $0.1 \mathrm{gr} / \mathrm{cm}^{3}$ intervals. The variogram of the Bouguer anomaly with $\rho=$ $2.3 \mathrm{gr} / \mathrm{cm}^{3} 3 \mathrm{gr} / \mathrm{cm} 3$ is exhibited, and linear property is presented while the sampling distances $27.5 \mathrm{~m}$ to $166 \mathrm{~m}$ is visible (Fig. 8).

This fractal behavior indicates the close relationship of the Bouguer gravity with topography. At the longer distances the nonlinear relationship and no fractal behavior is attributed to isostatic effects becoming dominate (Chapin 1996). Increasing the sampling interval, we will face longer wavelength.

The variogram of the fractal dimension versus the density and superimpose a least-squares line fitted into the curve has been indicated in Fig.9. This straight line is the reflection of the linear effect of the scale-dependence term. Therefore, removal the initial fractal dimension values from the computed fractal dimension values using least-squares line equation yields results that show a unique and optimum density that downgrades the topographic effects (Fig.10). The minimum value of the fractal dimension difference depends on $2.3 \mathrm{gr} / \mathrm{cm}^{3}$ density.

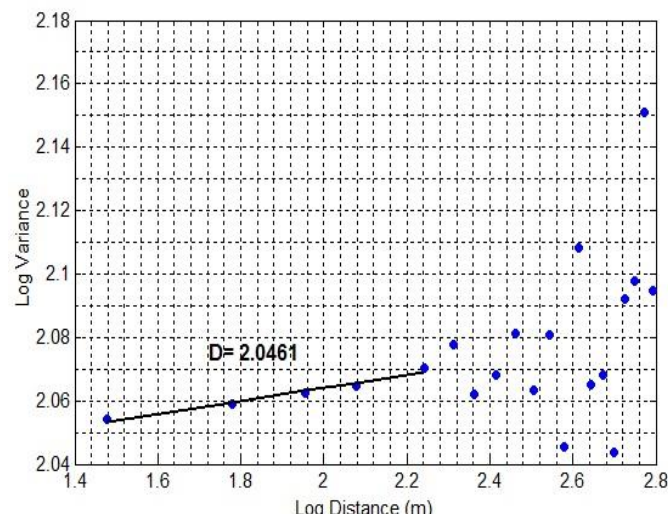

Fig. 8: Variogram for the Bouguer Anomaly with $2.3 \mathrm{Gr} / \mathrm{Cm}^{3}$ Density in the Mazandaran Area; Linear Feature Is for Sampling Distances between Approximately 27.5 and 166 M, But Nonfractal Outside that Range.

\section{Discussion and conclusions}

Requisite condition for density determination using the method that proposed by Thorarinsson and Magnusson (1990) delineate a circle as include the area maximum under consideration while mostly field operations is performed in the regions with nongeometric shape or lengthy regions for the 2.5 dimension targets.

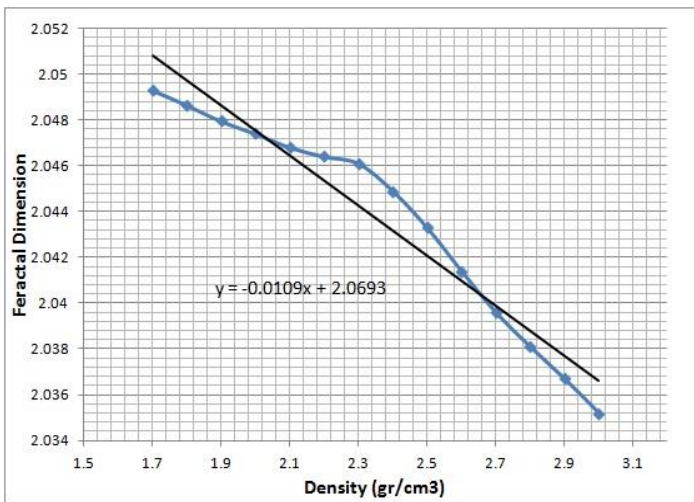

Fig. 9: Plot of the Feractal Dimension Versus Density for 14 Classes of the Bouguer Grids Computed Using Different Bouguer Densities from the
Mazandaran Gravity Data Set. The Linear Trend Is Caused by the Influence of the Linear Bouguer Slab Equation. In other Word, The ScaleDependence of the Ap Term Manifests Itself as a Linear Effect.

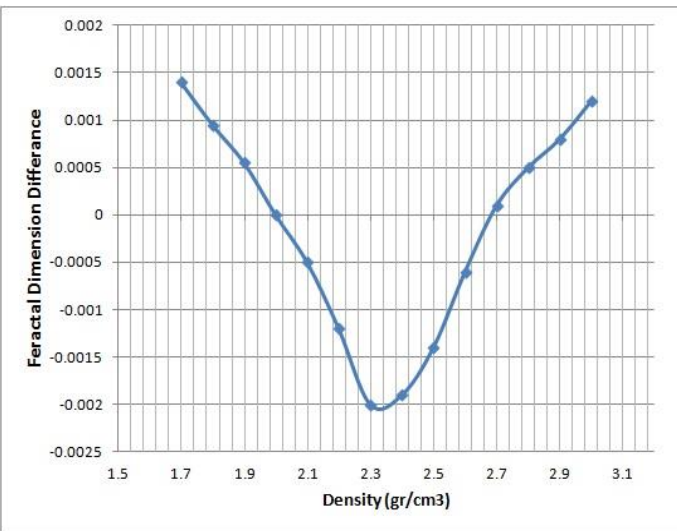

Fig. 10: Plot of the Fractal Dimension Differences versus the Density for 14 Classes of Different Bouguer Densities. The Effects of Topography and Bouguer Slab are minimized at $2.3 \mathrm{Gr} / \mathrm{Cm}^{3}$ Density. In other Word, This Density Is Optimum Density for Bouguer Correction.

One of the prominent advantages the mentioned method is the efficiency for all samplings designing in an exploratory area with various forms. Topography scale-independent factor in the Bouguer slab equation camber the drawn statistical variables versus the density. The linear trend is caused by the influence of the scale-dependence term of the Bouguer slab equation. The least difference between the two density functions yields the best density to use in the Bouguer slab correction. The dimension $\mathrm{D}$ of a fractal surface lies between 2D (a plane) and 3D (a solid volume) (Mandelbrot, 1975). In the proposed equation for the fractal dimension calculation, for zero degree tilt, the fractal dimension assumes two. When the diverse gravity data set was examined, the slope least-squares regression line in the variogram variance versus the distance would not be major of 0.3. All four statistical methods exhibit same consequences for the area under survey. The optimum density of $2.3 \mathrm{gr} / \mathrm{cm}^{3}$ shows a good conformity with the geological formation in Mazandaran region and is the best density that reduces all the data.

\section{References}

[1] Bichara M, Lakshmanan L (1983) Determination directe des densites du sol et de remblais a partir de mesures gravimetriques. Bull. lnt. Assoc. Eng. Geol., 26, 171-173 (in French with English abstract).

[2] Chapin A (1996) A deterministic approach toward isostatic gravity residuals - a case study from South America, Geophysics, 4, 10221033. http://dx.doi.org/10.1190/1.1444024.

[3] Fukao Y, Yamamoto A, Nozaki K (1981) A method of density determination for gravity correction. 1 Phys. Earth, 29, 163-166.

[4] Gibb R.A, Thomas M.D (1980) Density determinations of basic volcanic rocks of the Yellowknife supergroup by gravity measurements in mine shafts-Yellowknife, Northwest Territories. Geophysics, 45, 18-31. http://dx.doi.org/10.1190/1.1441036

[5] Grant F.S, Elsaharty A.F (1962) Bouguer gravity correction using a variable density. Geophysics, 5, 616-626. http://dx.doi.org/10.1190/1.1439071.

[6] Hammer S (1950) Density determinations by underground gravity measurements. Geophysics, 637-652. http://dx.doi.org/10.1190/1.1437625.

[7] Korvin G (1992) Fractal models in the earth sciences: Elsevier Science Publishers.

[8] LaFehr T.R (1983) Rock densities from borehole gravity surveys. Geophysics, 48, 341-356. http://dx.doi.org/10.1190/1.1441472.

[9] Lovejoy S, Schertzer S, Ladoy P (1986) Fractal characterization of homogeneous geophysical measuring network: Nature, 319, 43-44. http://dx.doi.org/10.1038/319043a0.

[10] [10] Mandelbrot, B. B., (1975) Stochastic models of the Earth's relief,the shape and the fractal dimension of the coastlines, and the number-area rule for islands: Proc. Nat. Acad. of Sci., 72, 3825-3828. http://dx.doi.org/10.1073/pnas.72.10.3825 
[11]Moribayashi S (1990) A new method for variable density correction of gravity data. BUTSURI-TANSA (Geophys. Explor.), 43, 97-106 (in Japanese with English abstract).

[12]Murata Y (1993) Estimation of optimum average surficial density from gravity data: An objective Bayesian approach. J. Geophys. Res., 98, 12097-12109. http://dx.doi.org/10.1029/93jb00192.

[13]Nettleton L.L (1939) Determination of density for the reduction of gravimeter observations.Geophysics, 4, 176-183. http://dx.doi.org/10.1190/1.1437088.

[14] Parasnis D.S (1952) A study of rock density in the English Midlands. Mon. Not. R. Astron. Soc. Geophys. Supp! 6, 252-271.

[15]Parasnis D.S (1979) Principles of aPPlied geophysics. Third edition, Chapman and Hall, London, 275 pp. http://dx.doi.org/10.1007/978-94009-5814-2.

[16] Rikitake T, Tajima H, Izutuya S, Hagiwara Y, Kawada K, Sasai Y (1965) Gravimetric and geomagnetic studies of Onikobe area. Bul!. Earthq. Res. Inst., Univ. Tokyo, 43, 241-267.

[17]Rimbert F, Erling j.c, Lakshmanan j (1987) Variable density Bouguer processing of gravity data from Herault, France. First Break, 5, 9-13. http://dx.doi.org/10.3997/1365-2397.1987001.

[18] Sissons, B.A (1981) Densities determined from surface and subsurface gravity measurements. Geophysics, 46, 1568-1571. http://dx.doi.org/10.1190/1.1441163.

[19] Thorarinsson F, Magnusson S. G (1990) Bouguer density determination by fractal analysis: Geophysics, 55, 932-935. http://dx.doi.org/10.1190/1.1442909.

[20]Vajk, R (1956) Bouguer corrections with varying surface density Geophysics, 4, 1004-1020. http://dx.doi.org/10.1190/1.1438292.

[21]Yamamoto A (1998) Estimating the Optimum Reduction Den-sity for Gravity Anomaly: A Theoretical Overview. Jour. Fac. Sci., Hokkaido Univ., Ser. VII (Geophysics), Vol. 11, No.3, 577-599, 1999. 